\title{
INVERSION OF AN INTEGRAL TRANSFORM ${ }^{1}$
}

\section{HARRY POLLARD AND HAROLD WIDOM}

1. Introduction. It is the object of this note to give an inversion formula for the integral transform,

$$
f(x)=\int_{0}^{\infty} k(y) \phi(x+y) d y, \quad x>0
$$

under the assumptions:

(i) $k \in L(0, \infty)$;

(ii) $k \neq 0$ in the neighborhood of zero;

(iii) the Laplace transform

$$
K(s)=\int_{0}^{\infty} e^{-8 x} k(x) d x
$$

has no zeros in the closed right half-plane;

(iv) $\phi \in L_{p}(0, \infty)$ for some $p$ in $1 \leqq p \leqq 2$.

Formally such a formula can be obtained as follows. Define $\phi$ and $k$ to be zero for negative values of the argument, and define $f$ by the equation in (1) for all real $x$. If we denote Fourier transformation by a circumflex, i.e.

$$
\hat{g}(t)=\int_{-\infty}^{\infty} e^{i t x} g(x) d x
$$

it follows from (1) that

$$
\hat{f}(t)=\hat{k}(-t) \widehat{\phi}(t) .
$$

Hence

$$
\begin{aligned}
\phi(x) & =\frac{1}{2 \pi} \int_{-\infty}^{\infty} e^{-i t x} \widehat{\phi}(t) d t=\frac{1}{2 \pi} \int_{-\infty}^{\infty} e^{-i t x} \frac{\hat{f}(t)}{\hat{k}(-t)} d t \\
& =\frac{1}{2 \pi} \int_{-\infty}^{\infty} e^{-i t x} \frac{d t}{\hat{k}(-t)} \int_{-\infty}^{\infty} e^{i t y} f(y) d y \\
& =\frac{1}{2 \pi} \int_{-\infty}^{\infty} f(x+y) d y \int_{-\infty}^{\infty} \frac{e^{i t y}}{\hat{k}(-t)} d t .
\end{aligned}
$$

Presented to the Society April 18, 1958 under the title Inversion of a class of convolutions; received by the editors January 2, 1958 and, in revised form, February 17, 1958.

1 This research was supported by the United States Air Force under Contract No. Af18(600)-685 monitored by the Office of Scientific Research. 
This last formula is clearly unsatisfactory since $f(x)$ is given only for $x>0$. However under our assumptions on $k$ it happens that

$$
\int_{-\infty}^{\infty} \frac{e^{i t y}}{\hat{k}(-t)} d t=0, \quad y<0,
$$

so the formula becomes

$$
\phi(x)=\frac{1}{2 \pi} \int_{0}^{\infty} f(x+y) d y \int_{-\infty}^{\infty} \frac{e^{i t y}}{\hat{k}(-t)} d t .
$$

The actual results which we shall prove, motivated by this formula, are these

TheOREM 1. Under hypotheses (i)-(iv) the equation (1) is inverted by

$$
\phi(x)=\underset{\delta \rightarrow 0+}{\operatorname{li}(p)} \lim _{\epsilon \rightarrow 0+} \frac{1}{2 \pi} \int_{0}^{\infty} f(x+y) d y \int_{-\infty}^{\infty} \frac{e^{i t(y-\delta)-\epsilon|t|}}{\hat{k}(-t)} d t .
$$

THEOREM 2. Under hypotheses (i)-(iv) the equation (1) is inverted by

$$
\phi(x)=\lim _{\delta \rightarrow 0+} \lim _{\epsilon \rightarrow 0+} \frac{1}{2 \pi} \int_{0}^{\infty} f(x+y) d y \int_{-\infty}^{\infty} \frac{1-e^{-i \delta t}}{i \delta t} \frac{e^{i t y-\epsilon|t|}}{\hat{k}(-t)} d t,
$$

for almost all $x$, and at all points of right-continuity of $\phi$.

An alternative technique, suggested by Sparenberg [3], seems difficult to apply.

2. Lemmas. We need Hayman's extension [1, Theorem 2] of the Ahlfors-Heins principle. ${ }^{2}$

Lemma 1. If $u(z)$ is subharmonic in the half-plane $y>0$ and if

$$
\begin{aligned}
\limsup _{z \rightarrow x} u(z) & \leqq 0, \\
\alpha & =\sup _{y>0} \frac{u(x+i y)}{y} \\
\alpha_{0} & =\limsup _{r \rightarrow \infty} \frac{1}{r} \sup _{|z|=r ; y>0} u(z)<\infty
\end{aligned}
$$

then $\alpha_{0}=\max (\alpha, 0)$ and $\lim u\left(r e^{i \theta}\right) / r=\alpha \sin \theta$ uniformly for $0<\theta<\pi$ as $r \rightarrow \infty$ omitting an $r$-set of finite logarithmic length (i.e., a set $E$ with $\left.\int_{E^{-1}}{ }^{-1} d r<\infty\right)$.

2 The authors are indebted to Professor W. H. J. Fuchs for calling their attention to this lemma, basic for their results. 
Lemma 2. Under the hypotheses (i)-(iii) imposed on $k$ the function $1 / \hat{k}(z)$ has the property

$$
\frac{1}{\hat{k}(z)}=O\left(e^{|z|}\right), \quad \quad \epsilon>0
$$

on a sequence of semi-circular arcs $|z|=r_{n}, 0 \leqq \theta \leqq \pi$, with $r_{n} \rightarrow \infty$ as $n \rightarrow \infty$.

Note that $\hat{k}(z)=K(-i z)$ so that $1 / \hat{k}(z) \neq 0$ for $y \geqq 0$. We may suppose that $|\hat{k}(z)| \leqq 1$ for $y \geqq 0$.

It follows from hypotheses (i)-(iii) that $\hat{k}$ has the representation

$$
u(z)=\log |\hat{k}(x+i y)|=\frac{y}{\pi} \int_{-\infty}^{\infty} \frac{\log |\hat{k}(v)|}{y^{2}+(x-v)^{2}} d v
$$

for $y>0$. See, for example, Nyman [2, pp. 13, 29].

Since $\log |\hat{k}(v)| \leqq 0$ it follows from the representation that $u(z) \leqq 0$ and also that $\lim _{y \rightarrow \infty} u(z) / y=0$. This confirms hypothesis (a) of Lemma 1 and shows that $\alpha=0$. (b) is also a consequence of the representation.

Hence

$$
\lim _{r \rightarrow \infty} \frac{u\left(r e^{i \theta}\right)}{r}=0
$$

with the prescribed uniformity. This establishes the lemma.

Lemma 3. Under the hypotheses (i)-(iii) $\lim _{u \rightarrow \infty} \log |\hat{k}(i u)| / u=0$.

This is an immediate consequence of the representation of $u(z)$.

3. The inversion formulas. It follows from (1) and the hypotheses (i), (iv) that

$$
\hat{f}(t)=\hat{k}(-t) \widehat{\phi}(t)
$$

where the Fourier transforms are taken in the appropriate sense. Therefore (by imitating the proof of Theorem 59 in Titchmarsh [4]) we have

$$
\phi(x)=\lim _{\epsilon \rightarrow 0+} \phi_{\epsilon}(x)
$$

where

$$
\phi_{\epsilon}(x)=\frac{1}{2 \pi} \int_{-\infty}^{\infty} e^{-i t x} e^{-\epsilon|t|} \frac{\hat{f}(t)}{\hat{k}(-t)} d t .
$$

Hence 


$$
\begin{aligned}
\phi_{\epsilon}(x) & =\frac{1}{2 \pi} \int_{-\infty}^{\infty} \frac{e^{-i t x} e^{-\epsilon|t|}}{\hat{k}(-t)} d t \underset{a \rightarrow \infty}{\operatorname{lq} . \mathrm{m} .} \int_{-a}^{a} e^{i t y} f(y) d y \\
& =\lim _{a \rightarrow \infty} \frac{1}{2 \pi} \int_{-\infty}^{\infty} \frac{e^{-i t x} e^{-\epsilon|t|}}{\hat{k}(-t)} d t \int_{-a}^{a} e^{i t y} f(y) d y \\
& =\lim _{a \rightarrow \infty} \frac{1}{2 \pi} \int_{-a}^{a} f(y) d y \int_{-\infty}^{\infty} \frac{e^{i t(y-x)} e^{-\epsilon|t|}}{k(-t)} d t \\
& =\frac{1}{2 \pi} \int_{-\infty}^{\infty} f(x+y) d y \int_{-\infty}^{\infty} \frac{e^{i t y} e^{-\epsilon|t|}}{\hat{k}(-t)} d t,
\end{aligned}
$$

where $q=p /(p-1)$. (2) is justified since $e^{-\epsilon|t|} / \hat{k}(-t)$ is in $L_{p}(-\infty, \infty)$, (3) by Fubini's theorem, and (4) because the inner integral as a function of $y$ is in $L_{q}(-\infty, \infty)$.

Now let

$$
R_{\epsilon}(y)=\int_{-\infty}^{\infty} \frac{e^{i t y-\epsilon|t|}}{\hat{k}(-t)} d t
$$

and consider the integral of

$$
\frac{e^{i w y-\epsilon w}}{\hat{k}(-w)}
$$

as a function of $w$, over the contour consisting of the intervals $(0, R)$ and $(0,-i R)$ and the quadrant of the circle joining $R$ and $-i R$. If $y<0$ it follows by Lemma 2 that the integral over the quadrant approaches zero as $R=r_{n} \rightarrow \infty$. Therefore

$$
\int_{0}^{\infty} \frac{e^{i t y-\epsilon t}}{\hat{k}(-t)} d t=\lim _{n \rightarrow \infty} \int_{0}^{r_{n}} \frac{e^{u y+i \epsilon u}}{\hat{k}(i u)} d u
$$

Similarly

$$
\int_{-\infty}^{0} \frac{e^{i t y+\epsilon t}}{\hat{k}(-t)} d t=-\lim _{n \rightarrow \infty} \int_{0}^{\tau_{n}} \frac{e^{u y-i \epsilon u}}{\hat{k}(i u)} d u .
$$

Thus for $y<0$

$$
R_{\epsilon}(y)=2 i \lim _{n \rightarrow \infty} \int_{0}^{r_{n}} \frac{e^{u y} \sin \epsilon u}{\hat{k}(i u)} d u=2 i \int_{0}^{\infty} \frac{e^{u y} \sin \epsilon u}{\hat{k}(i u)} d u .
$$

The last step is justified by Lemma 3 which enables us to conclude also that

$$
\underset{\lim . \operatorname{m}}{\log } R_{\epsilon}(y)=0 \quad \text { on }(-\infty,-\delta)
$$


for each $\delta>0$. According to (4)

$$
\phi(x)=\lim _{\epsilon \rightarrow 0+} \frac{1}{2 \pi} \int_{-\infty}^{\infty} f(x+y) R_{\epsilon}(y) d y
$$

and so

$$
\phi(x)=\lim _{\epsilon \rightarrow 0+} \frac{1}{2 \pi} \int_{-\delta}^{\infty} f(x+y) R_{\epsilon}(y) d y
$$

for each $\delta>0$. Consequently for each $\delta>0$

$$
\phi(x+\delta)=\lim _{\epsilon \rightarrow 0}+\frac{1}{2 \pi} \int_{0}^{\infty} f(x+y) R_{\epsilon}(y-\delta) d y
$$

and Theorem 1 follows.

We turn to the proof of Theorem 2. We have (by imitating the proof of Theorem 19 of Titchmarsh [4]) $\phi(x)=1$. i.m. $\stackrel{(p)}{\leftrightarrow \rightarrow+} \phi_{\epsilon}(x)$, so for any fixed $x, \phi(x+h)=1$.i.m. $\stackrel{(p)}{\leftrightarrow 0+} \phi_{\epsilon}(x+h)$ over any $h$-interval $(0, \delta)$. Therefore, by the argument leading to (5),

$$
\phi(x+h)=\underset{\epsilon \rightarrow 0+}{\stackrel{(1)}{1} .} \frac{1}{2 \pi} \int_{0}^{\infty} f(x+y) R_{\epsilon}(y-h) d y,
$$

so

$$
\begin{aligned}
\int_{0}^{\delta} \phi(x+h) d h & =\lim _{\epsilon \rightarrow 0+} \frac{1}{2 \pi} \int_{0}^{\delta} d h \int_{0}^{\infty} f(x+y) R_{\epsilon}(y-h) d y \\
& =\lim _{\epsilon \rightarrow 0+} \frac{1}{2 \pi} \int_{0}^{\infty} f(x+y) d y \int_{0}^{\delta} R_{\epsilon}(y-h) d h \\
& =\lim _{\epsilon \rightarrow 0+} \frac{1}{2 \pi} \int_{0}^{\infty} f(x+y) d y \int_{-\infty}^{\infty} \frac{1-e^{i \delta t}}{i \delta t} \frac{e^{i t y-\epsilon|t|}}{\hat{k}(-t)} d t
\end{aligned}
$$

and Theorem 2 follows.

\section{REFERENCES}

1. W. K. Hayman, Questions of regularity connected with the Phragmen-Lindelöf principle, J. Math. Pures Appl. vol. 35 (1956) pp. 115-126.

2. B. Nyman, On the one-dimensional translation group and semi-group in certain function spaces, Upsala, 1950.

3. J. A. Sparenberg, Application of the theory of sectionally holomorphic functions to Wiener-Hopf type integral equations, Nederl. Akademie Wetensch. Proceedings Ser. A. vol. 59 (1956) pp. 29-34.

4. E. C. Titchmarsh, Introduction to the theory of Fourier integrals, Oxford, 1937

CORNELl University 